\title{
Increased Resistance of Skin Flora to Antimicrobial Prophylaxis in Patients Undergoing Hip Revision Arthroplasty
}

\author{
HEINRICH M.L. MÜHLHOFER, LUKAS DEISS, PHILIPP MAYER-KUCKUK, FLORIAN POHLIG, \\ NORBERT HARRASSER, ULRICH LENZE, HANS GOLLWITZER, CHRISTIAN SUREN, \\ PETER PRODINGER, RÜDIGER VON EISENHART-ROTHE and JOHANNES SCHAUWECKER
}

\author{
Department of Orthopaedic Surgery, Klinikum rechts der Isar, Technical University Munich, Munich, Germany
}

\begin{abstract}
Background/Aim: Prosthetic joint infection (PJI) remains a major complication after total joint replacement and is the primary indication for revision arthroplasty. Specifically, coagulase-negative Staphylococci (CNS) can cause low-grade infections. Despite the use of cephalosporin-based antimicrobial prophylaxis (AMP) and antiseptic treatment at the surgical site, evidence suggests that a significant number of cases of dermal CNS results in low-grade PJI. Thus, this study examined the bacterial colonization and resistance patterns at the surgical site. We hypothesized that the bacteria developed resistance to antibiotics that are frequently used in primary and revision total hip arthroplasty (THA) procedures. Patients and Methods: Ninety patients, including 63 primary and 27 revision THA patients, were enrolled in this study. For each patient, a single swab of the skin at the surgical site was subjected to clinical microbiology to assess bacterial colonization. Furthermore, resistance to a sentinel panel of antibiotics (benzylpenicillin, erythromycin, tetracycline, oxacillin, fusidic acid, clindamycin, gentamicin, levofloxacin/ moxifloxacin, rifampicin, linezolid and vancomycin) was tested. Results: In $96.7 \%$ of the patients, at least one bacterial strain was identified at the surgical site, with CNS strains comprising $93.1 \%$ of the total. The sentinel panel showed that $30.7 \%$ of the CNS strains exhibited maximal resistance to oxacillin, a commonly used cephalosporin. Additionally, oxacillin resistance increased 1.9-fold $(p=0.042)$ between primary and revision THA. Notably, $8.1 \%$ of the CNS stains found on
\end{abstract}

This article is freely accessible online.

Correspondence to: Heinrich Mühlhofer, MD, Department of Orthopaedic Surgery, Ismaninger Str. 22, 81675 Munich, Germany. Tel: +49 8941405490, Fax: +49 8941404849, e-mail: heinrich. muehlhofer@mri.tum.de

Key Words: Prosthetic joint infection, antimicrobial prophylaxis, coagulase-negative Staphylococci. patients undergoing primary THA were resistant to gentamicin, an aminoglycoside, and this rate increased 4.7-fold ( $p=0.001)$ for patients undergoing revision THA. Conclusion: CNS strains have significant resistance to standard AMP, particularly in individuals undergoing revision THA.

Prosthetic joint infection (PJI) remains a major complication after total joint replacement and is the primary indication for revision arthroplasty (1). Data have shown that revisions due to PJI compose approximately $25 \%$ of all revision total hip arthroplasty (THA) procedures (2). The challenge in controlling PJI is underscored by projections suggesting a robust increase in THA over the next 15 years (3). The rapid development of PJI shortly after surgery is typically caused by highly virulent bacteria, such as Staphylococcus aureus, and associated with acute symptoms, such as pain and fever. In contrast, late manifestations of PJI are often due to a lowgrade infection with less virulent bacterial strains of the dermal flora, including coagulase-negative Staphylococci (CNS). These low-grade PJI infections frequently result in septic loosening of the prosthetic components over time (4). Although the origin of the microorganisms causing either acute- or low-grade infections cannot be precisely identified in all cases, studies have strongly suggested that intraoperative contamination (nosocomial) either at the surgical site or on the prosthetic components with bacteria from the patient's skin causes PJI $(1,5,6,7)$ despite prophylactic treatment of the skin with antiseptic agents. This is probably because topical antiseptics have limited effectiveness against bacteria in the deeper layers of the stratum corneum (8). Infections at the surgical site occur in approximately $1-1.5 \%$ of primary THA cases and there is evidence of a higher rate in patients undergoing revision THA (9-11).

To prevent nosocomial surgical site infections, antimicrobial prophylaxis (AMP) was introduced in the 1980s $(12,13)$. Current AMP protocols are based on firstgeneration cephalosporins in the US and second- and thirdgeneration cephalosporins in Europe (14). Despite the use of 
AMP, patient data collected in the US between 2001 and 2009 showed an increase in the annual incidence of PJI from $2.05 \%$ to $2.18 \%(15,16)$. One potential reason for this observation is that AMP has become increasingly ineffective against strains that commonly cause PJI. This view is supported by the finding that cephalosporin-resistant strains have been detected in patients with PJI after primary joint arthroplasty (17). Previous work on patient cohorts receiving oxacillin-based AMP analysed bacterial susceptibility of nose or groin swabs collected before and after joint revision (2week period) and showed increases in resistance to isoxazolyl, penicillin, clindamycin, fusidic acid and gentamicin (18). These data support historical observations that nostril swabs obtained from patients two weeks after THA contained an increasing number of bacteria resistant to methicillin, tetracycline, erythromycin, clindamycin and gentamicin than swabs taken one day prior to surgery (19). Based on these findings, we hypothesized that patients undergoing revision THA are colonized with bacteria with an increased resistance to current cephalosporin-based AMP; therefore, we compared resistance to a panel of 12 antibiotics, including the cephalosporin sentinel oxacillin, in patients undergoing primary and revision arthroplasty.

\section{Patients and Methods}

A total of 90 consecutive patients (48 men and 42 women) undergoing either primary arthroplasty or aseptic revision arthroplasty at the Department of Orthopaedic Surgery, Klinikum rechts der Isar, Technische Universität München, München, Germany, were included in this prospective study. The age of the patients ranged between 22 and 85 years with a mean patient age of $65 \pm 14.7$ years. Osteoarthritis was the primary diagnosis for all patients undergoing primary THA, with aseptic loosening being the primary diagnosis for the revision surgery. None of the patients received antibiotics within three months prior to surgery and no local antiseptic treatment, such as chlorhexidine decolonization, was provided. Patients received a single $1.5 \mathrm{~g}$ dose of the secondgeneration cephalosporin cefuroxime as their AMP. Primary and revision THA was performed on 63 and 27 patients, respectively. For primary THA, a direct anterior approach $(n=37)$ or an anterolateral approach $(n=26)$ was used, whereas all revision THA procedures exclusively used the antero-lateral technique.

Samples were collected the morning before surgery based on established procedures (18). In brief, a single culture sample was obtained with a sterile cotton swab of the skin at the planned surgical site. Standard clinical microbiology was performed at the Department of Microbiology at our Hospital. The swabs were then applied to aerobic agar plates (Columbia sheep blood agar, chocolate agar, MacConkey agar) (Becton Dickinson Gmbh, Heidelberg, Germany) and incubated in an aerobic atmosphere at $37^{\circ} \mathrm{C}$ for $48 \mathrm{~h}$, after which the bacterial strains were identified using a matrix-assisted laser desorption/ionization time-of-flight (MALDITOF) mass spectrometer (Bruker Daltonik, Bremen, Germany). Antimicrobial susceptibility to the antibiotics benzylpenicillin, erythromycin, tetracycline, oxacillin, fusidic acid, clindamycin, gentamicin, levofloxacin/moxifloxacin, rifampicin, linezolid and
Table I. Resistance rates.

\begin{tabular}{lccc}
\hline Sentinel antibiotics & $\begin{array}{c}\text { Class of } \\
\text { anitbiotic }\end{array}$ & $\begin{array}{c}\text { Resistance } \\
\text { rate }(\%)\end{array}$ & 95\%-CI \\
\hline Benzylpenicillin & Penicillin & 77.3 & $70.6-84$ \\
Erythromycin & Macrolide & 58 & $50.1-65.9$ \\
Tetracycline & Tetracycline & 42 & $34-49.9$ \\
Oxacillin & Cephalosporin & 34.7 & $27.1-42.3$ \\
Fusidic acid & Steroid antibiotics & 32 & $24.5-39.5$ \\
Clindamycin & Lincosamide & 28.7 & $21.4-35.9$ \\
Gentamicin & Aminoglycoside & 15.3 & $9.6-21.1$ \\
Levofloxacin & Fluocinolone & 14 & $8.4-19.6$ \\
Moxifloxacin & Fluocinolone & 14 & $8.4-19.6$ \\
Rifampicin & Ansamycin & 2 & $0-4.2$ \\
Linezolid & Oxazolidinone & 0.7 & $0.001-2$ \\
Vancomycin & Glycopeptide & 0 & n.p. \\
\hline
\end{tabular}

CI, Confidence interval; n.p., not possible.

vancomycin was determined using a VITEK 2 XL (bioMerieux, Nürtingen, Germany). Resistance patterns of the dermal bacteria from patients undergoing primary arthroplasty were compared to those of patients undergoing revision arthroplasty using SPSS software (SPSS Inc., Chicago, IL, USA) and Pearson's Chi-square test. Furthermore, the absolute and relative frequencies, as well as the confidence intervals (CIs) were calculated.

\section{Results}

Among the 90 patients analysed, $96.7 \%$ had at least one strain on the skin at the surgical site. The detected bacteria comprised 144 strains; CNS strains were the most frequent strains at a rate of $93.1 \%$. To determine the antibiotic susceptibility of the CNS strains, we used a panel of 12 antibiotics according to the standard microbiological diagnostics at our Institution. The highest resistance rate was observed for benzylpenicillin (77.3\%, Table I), which was an expected result (20). More importantly, we observed substantial resistance to several of the antibiotics frequently employed in the management of orthopaedic patients. For example, $34.7 \%, 28.7 \%$ and $15.3 \%$ of the CNS strains displayed resistance to oxacillin, clindamycin and gentamicin, respectively (Table I); notably, no vancomycinresistant strains were identified. A comparison between patients undergoing primary versus revision THA revealed a significant increase in the resistance to four of the 12 tested antibiotics (Table II). Oxacillin and gentamicin demonstrated 1.9-fold and 4.7-fold increases in resistance, respectively, which corresponded to $46.2 \%$ and $38.5 \%$ of patients undergoing revision THA. As oxacillin is a commonly used cephalosporin, these data suggest the limited efficacy of standard cephalosporin-based AMP currently employed in the US and Europe. 
Table II. Comparison of antibiotic susceptibility of CNS strains between primary and revision THA.

\begin{tabular}{|c|c|c|c|c|c|c|c|}
\hline \multirow[b]{2}{*}{ Sentinel } & \multicolumn{2}{|c|}{ Primary THA } & \multirow[b]{2}{*}{ Sentinel } & \multicolumn{2}{|c|}{ Revision THA } & \multicolumn{2}{|c|}{ Comparison } \\
\hline & Rate & $95 \%-\mathrm{CI}$ & & Rate & $95 \%-\mathrm{CI}$ & $p$-Value & Chi-square \\
\hline Oxacillin & 24.2 & $13.5-34.9$ & Oxacillin & 46.2 & $27-65.3$ & $\mathrm{p}<0.05$ & 4.154 \\
\hline Benzylpenicillin & 72.6 & $61.5-83.7$ & Benzylpenicillin & 84.6 & $70.7-98.5$ & $p>0.05$ & 1.46 \\
\hline Gentamicin & 8.1 & $1.3-14.8$ & Gentamicin & 38.5 & $19.8-57.2$ & $p<0.01$ & 11.97 \\
\hline Levofloxacin & 8.1 & $1.3-14.8$ & Levofloxacin & 26.9 & $9.9-44$ & $p<0.05$ & 5.532 \\
\hline Moxifloxacin & 8.1 & $1.3-14.8$ & Moxifloxacin & 26.9 & $9.9-44$ & $p<0.05$ & 5.532 \\
\hline Erythromycin & 56.5 & $44.1-68.8$ & Erythromycin & 61.5 & $42.8-80.2$ & $p>0.05$ & 0.195 \\
\hline Clindamycin & 29 & $17.7-40.3$ & Clindamycin & 34.6 & $16.3-52.9$ & $p>0.05$ & 0.268 \\
\hline Vancomycin & 0 & n.p. & Vancomycin & 0 & n.p. & n.p. & n.p. \\
\hline Tetracycline & 25.8 & $14.9-36.7$ & Tetracycline & 34.6 & $16.3-52.9$ & $p>0.05$ & 0.699 \\
\hline Linezolid & 1.6 & $0.0-4.7$ & Linezolid & 0 & n.p. & $p>0.05$ & 0.424 \\
\hline Rifampicin & 1.6 & $0.0-4.7$ & Rifampicin & 7.7 & $0.0-17.9$ & $p>0.05$ & 2.056 \\
\hline Fusidic acid & 32.3 & $20.06-43.09$ & Fusidic acid & 38.5 & $19.8-57.2$ & $p>0.05$ & 0.314 \\
\hline
\end{tabular}

CNS, Coagulase-negative Staphylococci; THA, total hip arthroplasty; CI, confidence interval; n.p., not possible.

\section{Discussion}

Our study led to three central findings. First, and perhaps most importantly, we demonstrated that approximately $50 \%$ of all patients undergoing revision THA are likely not to benefit from standard cephalosporin-based AMP due to resistance to cephalosporins. Second, we observed that approximately $40 \%$ and $30 \%$ of patients are resistant to gentamicin and clindamycin, respectively; these two antibiotics are frequently used in cemented THA to protect against infection $(21,22)$, an observation that has been reported in previous studies (18, 23). Finally, we noted a relatively low prevalence of methicillin-resistant Staphylococcus aureus (MRSA) (0.4\%), indicating that cephalosporin-based AMP is effective to prevent surgical side infections caused by $S$. aureus.

The considerably high rate of resistance to oxacillin was partially due to an increase in resistance between primary and revision THA. The underlying cause for this increase is currently unclear; however, two potential contributing factors should be discussed. First, bacterial colonization during the hospital stay may result in a higher probability of CNS strains on the skin of patients undergoing revision surgery. Several studies suggest a correlation between days of hospitalization and the presence of resistant CNS strains on the skin $(23,24)$. Indeed, the ward staff rather than the theatre staff or surgeons have been identified as a common source of oxacillin-resistant CNS strains (23). Therefore, patient exposure to the ward staff during their hospital stay is a risk for acquiring oxacillin-resistant CNS strains. Second, there is the possibility of the initial AMP selecting for oxacillin-resistant strains. To this end, it appears to be critical that hospitals strictly adhere to the guidelines for the proper use of AMP (25).
An important consideration is how to improve current AMP regimens and achieve lower infection rates in patients undergoing THA, especially those requiring a revision. Notably, our data suggest a role for vancomycin as we found no vancomycin-resistant strains during either primary or revision THA. However, some reports have raised concerns regarding the toxicity of glycopeptides due to the slow tissue distribution of vancomycin $(21,26,27)$. This notwithstanding, in our experience (Mühlhofer, unpublished data), administration of vancomycin $(10-15 \mathrm{mg} / \mathrm{kg}$ ) one to two hours prior to surgery yields adequate tissue concentrations with less than $1 \%$ nephrotoxicity after a single dose. Furthermore, due to its longer half-life, a second dose of vancomycin is typically unnecessary $(28,29)$. The use of AMP regimens for patients undergoing revision arthroplasty should account for CNS strains with potentially significant oxacillin resistance and short-term decolonization of the skin may provide additional value prior to revision arthroplasty.

\section{Ethics and Consent to Participate}

The present study was approved by the local ethics committee $(4092 / 11)$ and written consent was obtained from each subject prior to inclusion.

\section{Conflicts of Interest}

All Authors declare that they have no competing interests.

\section{Funding}

This study was not supported by extramural funding. 


\section{References}

1 Zimmerli W and Trampuz A: Prosthetic-joint infections. New Engl J Med 351(16): 1645-1654, 2004.

2 Havelin LI, Fenstad AM, Salomonsson R, Mehnert F, Furnes O, Overgaard S, Pedersen AB, Herberts P, Kärrholm J and Garellick G: The Nordic Arthroplasty Register Association. SORT 80: 393-401, 2009.

3 Kurtz S: Projections of primary and revision hip and knee arthroplasty in the United States from 2005 to 2030. J Bone Joint Surg Am 89: 780, 2007.

4 Maderazo EG, Judson S and Pasternak H: Late Infections of total joint prostheses: A review and recommendations for prevention. Clin Orthopaed Related Res 229: 131, 1988.

5 Shahi A and Parvizi J: Prevention of periprosthetic joint infection. Arch Bone Jt Surg 3: 72-81, 2015.

6 Lee J, Singletary R, Schmader K, Anderson DJ, Bolognesi M and Kaye KS: Surgical site infection in the elderly following orthopaedic surgery. Risk factors and outcomes. J Bone Joint Surg Am 88: 1705-1712, 2006.

7 Robert-Koch-Institut: Definition noskomialer Infektionen (CDCDefintionen), 7.Auflage, Berlin 2011. pp. 1-68, 2011.

8 Larson E: Guideline for use of topical antimicrobial agents. Am J Infect Control 16: 253-266, 1988.

9 Urquhart DM, Hanna FS, Brennan SL, Wluka AE, Leder K, Cameron PA, Graves SE and Cicuttini FM: Incidence and risk factors for deep surgical site infection after primary total hip arthroplasty: A systematic review. J Arthroplasty 25: 1216-22.e13, 2010.

10 Pugely AJ, Callaghan JJ, Martin CT, Cram P and Gao Y: Incidence of and risk factors for 30-day readmission following elective primary total joint arthroplasty: Analysis from the ACSNSQIP. J Arthroplasty 28: 1499-1504, 2013.

11 Song K-H, Kim ES, Kim YK, Jin HY, Jeong SY, Kwak YG, Cho YK, Sung J, Lee Y-S, Oh H-B, Kim TK, Koo K-H, Kim E-C, Kim JM, Choi TY, Kim HY, Choi HJ, Kim HB: KONIS Study Group: Differences in the risk factors for surgical site infection between total hip arthroplasty and total knee arthroplasty in the Korean nosocomial infections surveillance system (KONIS). Infect Control Hosp Epidemiol 33: 1086-1093, 2015.

12 Hill C, Flamant R, Mazas F and Evrard J: Prophylactic cefazolin versus placebo in total hip replacement. Report of a multicentre double-blind randomised trial. Lancet 1(8224): 795-796, 1981.

13 Doyon F, Evrard J, Mazas F and Hill C: Long-term results of prophylactic cefazolin versus placebo in total hip replacement. Lancet 1(8537): 860, 1987.

14 Alexiou VG, Ierodiakonou V, Peppas G and Falagas ME: Antimicrobial prophylaxis in surgery: An international survey. Surgical Infect 11: 343-348, 2010.

15 Tande AJ and Patel R: Prosthetic joint infection. Clin Microbiol Reviews 27: 302-345, 2014.

16 Kurtz SM, Lau E, Watson H, Schmier JK and Parvizi J: Economic burden of periprosthetic joint infection in the United States. J Arthroplasty 27: 61-65.e1, 2012.
17 Phillips JE, Crane TP, Noy M, Elliott TSJ and Grimer RJ: The incidence of deep prosthetic infections in a specialist orthopaedic hospital: A 15-year prospective survey. J Bone Joint Surg Br 88: 943-948, 2006.

18 Stefánsdóttir A, Johansson Å, Lidgren L, Wagner P and W-Dahl A: Bacterial colonization and resistance patterns in 133 patients undergoing a primary hip- or knee replacement in Southern Sweden. SORT 84: 87-91, 2013.

19 Sanzén L and Walder M: Antibiotic resistance of coagulasenegative staphylococci in an orthopaedic department. J Hosp Infect 12: 103-108, 1988.

20 Schoenfelder SMK, Dong Y, Feßler AT, Schwarz S, Schoen C, Köck R and Ziebuhr W: Antibiotic resistance profiles of coagulase-negative staphylococci in livestock environments. Vet Microbiol 200: 79-87, 2017.

21 Parvizi J, Saleh KJ, Ragland PS, Pour AE and Mont MA: Efficacy of antibiotic-impregnated cement in total hip replacement. SORT 79: 335-341, 2008.

22 Engesæter LB, Lie SA, Espehaug B, Furnes O, Vollset SE and Havelin LI: Antibiotic prophylaxis in total hip arthroplasty: Effects of antibiotic prophylaxis systemically and in bone cement on the revision rate of 22,170 primary hip replacements followed 0-14 years in the Norwegian Arthroplasty Register. Acta Orthop Scand 74: 644-651, 2003.

23 Sanzén L and Walder M: Antibiotic resistance of coagulasenegative staphylococci in an orthopaedic department. J Hosp Infect 12: 103-108, 1988.

24 Cruse P and Foord R: The epidemiology of wound infection. A 10-year prospective study of 62,939 wounds. Surg Clin North Am 60(1): 27-40, 1980.

25 Kreisel D, Savel TG, Silver AL and Cunningham JD: Surgical antibiotic prophylaxis and Clostridium difficile toxin positivity. Arch Surg 130: 989-993, 1995.

26 Evans RP: Current concepts for clean air and total joint arthroplasty: Laminar airflow and ultraviolet radiation: A Systematic review. Clin Orthopaed Related Res 469: 945-953, 2010.

27 Hedin G and Hambraeus A: Daily scrub with chlorhexidine reduces skin colonization by antibiotic-resistant Staphylococcus epidermidis. J Hosp Infect 24: 47-61, 1993.

28 Fletcher N: Prevention of perioperative infection. J Bone Joint Surg Am 89: 1605-1614, 2007.

29 Meehan J, Jamali AA and Nguyen H: prophylactic antibiotics in hip and knee arthroplasty. J Bone Joint Surg Am 91: 2480-2490, 2009. 